\title{
Fréquence des helminthes chez les volailles en Iran
}

\author{
par A.H. ESLAMI (*) et M. ANWAR (*)
}

\begin{abstract}
RESUME
Les auteurs ont systématiquement recherché les helminthes hébergés par cent poulets de ferme, de provenances diverses.

Ils donnent la liste des parasites rencontrés, en précisant ceux qui sont signalés pour la première fois en Iran.
\end{abstract}

Cent poulets provenant de différentes fermes ont été examinés pour recherche et détermination des helminthes présents dans les différents organes.

14 genres différents d'helminthes ont été mis en évidence: 5 Trématodes, 3 Cestodes et 6 Nématodes.

Les helminthes ci-dessous sont décrits pour la première fois en Iran.

Subulura brumpti, Gongylonema sp., Capillaria annulata, Capillaria caudinflata, Capillaria contorta, Prosthogonimus furcifer, Hypoderaeum conoideum, Raillietina ransomi et Hymenolepis carioca.

\section{INTRODUCTION}

Dans les sociétés rurales de l'Iran, l'élevage des poulets de ferme sous forme traditionnelle est très répandu. Ces modes d'élevage, l'absence de mesures appropriées pour en limiter les effets sont la cause d'une forte infestation parasitaire chez les volailles considérées.

Bien que cet élevage joue un rôle important dans l'économie rurale, il y a peu d'information sur les maladies parasitaires chez ces animaux. Seuls MIRZAYANS et NIAK (1) ont étudié

(*) Université de Téhéran, Faculté de Médecine Vétérinaire, Département de Parasitologie, B.P. 3262, Téhéran, Iran. la contamination des poulets de ferme par des Capillaria dans une petite région de l'Iran près de Téhéran.

\section{MATERIEL ET METHODE}

Les poulets examinés proviennent des différentes parties du pays. Dans cet ordre d'idée ont été examinés: le tissu sous-cutané, la trachée, l'appareil génital et le tube digestif.

Les organes digestifs sont ouverts au-dessus d'un tamis métallique possédant cent mailles par $25 \mathrm{~mm}^{2}$ et lavés sous l'eau courante jusqu'à ce qu'ils soient propres. Le contenu du tamis est examiné par petites quantités dans une boîte de Pétri placée sur un fond noir. Les parasites récoltés sont conservés dans de l'alcool à 70 p. 100; les grands Cestodes sont colorés avec de l'aceto-alun carmine. Les Nématodes et les Trématodes sont rendus transparents par le lacto-phenol afin de pouvoir procéder à leur identification. Lorsque le nombre des Capillaria ou des Heterakis est très élevé, c'est la méthode de dilution [méthode de TAYLOR (2)] qui est employée pour estimer le nombre de vers existant. Quand il y a également un grand nombre de différentes espèces de Capillaria, les espèces d'une petite partie sont identifiées et leur nombre total est estimé par calcul proportionnel. La même méthode est utilisée pour estimer le rapport du 
TABLEAU N $N^{\bullet}$ I

Nêmatodes collectés pour 100 poulets

\begin{tabular}{|c|c|c|c|c|c|c|}
\hline Es p èces & $\begin{array}{c}\text { Pourcentage } \\
\text { de } \\
\text { contamination }\end{array}$ & $\begin{array}{c}\text { Nombre total } \\
\text { des } \\
\text { vers collectés }\end{array}$ & $\begin{array}{l}\text { Intervalle } \\
\text { de } \\
\text { confiance }\end{array}$ & $\begin{array}{l}\text { Nombre moyen } \\
\text { des vers par } \\
\text { poulet }\end{array}$ & $\begin{array}{l}\text { Pourcentage } \\
\text { des mâles }\end{array}$ & $\begin{array}{c}\text { Pourcentage } \\
\text { des } \\
\text { femelles }\end{array}$ \\
\hline Heterakis gallinarum & 66 & 5.682 & $1-1000$ & 86 & 17,6 & 82,4 \\
\hline Ascaridia galzi & 44 & 418 & $1-70$ & 9,5 & 31,8 & 68,2 \\
\hline Subulura brumpti & 4 & 183 & $10-143$ & 46 & 28,4 & 71,6 \\
\hline Gongy Zonema sp. & 1 & 1 & 1 & 1 & - & $I$ \\
\hline Syngomis trached & 19 & 65 & $1-14$ & 3,4 & 50 & 50 \\
\hline Capiliaria sp. & 69 & 3.672 & $1-1000$ & 53,2 & 16,5 & 83,5 \\
\hline Capilzaria annulata & 1,14 & 42 & $1-34$ & - & - & - \\
\hline Capitzaria bursata & 52,8 & 1.940 & - & - & - & - \\
\hline Capilzaria anatis & 35,2 & 1.293 & - & - & - & - \\
\hline Capillaria axudinflata & 10,65 & 389 & - & - & - & - \\
\hline Capitiaria contonta & 0.08 & 3 & - & - & - & - \\
\hline Capillaria obsignata & 0,03 & 5 & - & - & - & - \\
\hline
\end{tabular}

*par rapport à la moyenne.

TABLEAU $\mathbb{N}^{\circ}$ II

Trématodes collectés pour 100 poulets

\begin{tabular}{|c|c|c|c|c|}
\hline Is p è c e s & $\begin{array}{l}\text { Pourcentage } \\
\quad \text { de } \\
\text { contamination }\end{array}$ & $\begin{array}{l}\text { Nombre rotal } \\
\text { des vers }\end{array}$ & $\begin{array}{c}\text { Intervalle } \\
\text { de } \\
\text { confiance }\end{array}$ & $\begin{array}{c}\text { Nombre moyen } \\
\text { des vers par } \\
\text { poulet }\end{array}$ \\
\hline Echinostona revolutum & 6 & 76 & $1-34$ & 12,7 \\
\hline Hypoderaeum conoideum & 1 & 1 & $I$ & 1 \\
\hline Prosthogonimus furcifer & 1 & 5 & 5 & 5 \\
\hline Echinoparypinim recurvatum & 1 & 3 & 3 & 3 \\
\hline Brachytaemus cormutatus & 1 & 1 & 1 & 1 \\
\hline
\end{tabular}

* par rapport à la moyenne.

TABLLASU N* J II

Cestodes collectes pour 100 poulets

\begin{tabular}{|c|c|c|c|c|}
\hline Es p è $\mathrm{ces}$ & $\begin{array}{l}\text { Pourcentage } \\
\text { de } \\
\text { contamination }\end{array}$ & $\begin{array}{l}\text { Nombre tot } 11 \\
\text { des vers }\end{array}$ & $\begin{array}{l}\text { Incervalde } \\
\text { de } \\
\text { cuntarance }\end{array}$ & $\begin{array}{c}\text { Numbre moyen } \\
\text { des vers par } \\
\text { poulet }\end{array}$ \\
\hline Raillietina eahinobothrida & 44 & 168 & $1-34$ & 3,8 \\
\hline Raillietina tetragona & 31 & 291 & $1-64$ & 9,6 \\
\hline Raillietina cesticillus & 6 & 24 & $1-12$ & 3 \\
\hline Railzietina ransomi & 8 & 194 & $1-94$ & 24,9 \\
\hline Railitetina magninumida & 3 & 3 & 1 & 1 \\
\hline Choanotaenia infundibutum & 2 & 5 & $1-3$ & 2,5 \\
\hline Bymenolepis carioca & 1 & 38 & 38 & 38 \\
\hline
\end{tabular}

* par rapport à la moyenne. 
nombre des mâles et des femelles des Nématodes.

\section{RESULTATS}

96 p. 100 des poulets examinés dans cette étude hébergeaient au moins une espèce d'helminthes. Le pourcentage de contamination par Nématodes, Cestodes et Trématodes a respectivement été de 96 p. 100,64 p. 100 et 10 p. 100.

Dans le tableau $n^{\circ} \mathrm{I}$, la proportion de contamination par les différentes espèces de Capillaria est donnée. Les Capillaria sont les Nématodes les plus abondants, ensuite ce sont les Heterakis gallinarum et les Ascaridia galli.

\section{DISCUSSION}

Les poulets examinés dans cette étude proviennent des différentes parties du pays. Il semble que la plupart des parasites décrits existent un peu partout en Iran. On peut donc utiliser les résultats de cette étude comme valables pour tout le pays.

Il n'est pas inutile de rappeler que la liste ci-dessous ne constitue qu'un premier recensement de ces parasites en Iran :

Subulura brumpti, Gongylonema sp., Capillaria annulata, Capillaria caudinflata, Capillaria contorta, Prosthogonimus furcifer, Hypoderaeum conoideum, Raillietina ransomi et Hymenolepis carioca.

ZARRINE (3) a démontré que 14,2 p. 100 des poulets élevés dans des conditions industrielles ont été contaminés par des Cestodes. La proportion est donc plus grande dans notre étude (64 p. 100). Nous avons également examiné 150 tubes digestifs de poulets provenant d'élevages industriels. La proportion de la contamination par les différents helminthes était de beaucoup inférieure à celle constatée chez les poulets de ferme puisqu'elle ne dépassait pas 1 p. 100.

ZARRINE a aussi démontré, comme dans notre étude, que Raillietina echinobothrida et Raillietina tetragona sont très communs chez les poulets industriels, ce qui signifierait que ces deux parasites sont les Cestodes les plus répandus chez les poulets en Iran.

En 1970, MIRZAYANS et NIAK ont étudié les Capillaria des poulets de ferme dans une certaine région de l'Iran. Ils ont remarqué que 26 p. 100 de poulets examinés étaient contaminés, alors que dans nos travaux la proportion de contamination est nettement plus forte $(69 \mathrm{p}$. 100).

Ces auteurs ont signalé seulement trois espèces de Capillaria. En outre, ils ont trouvé que Capillaria obsignata était plus répandu, alors que ce dernier est plus rare dans notre observation.

Bien que Capillaria sp. et Heterakis gallinarum soient nombreux chez les poulets examinés, nous n'avons pas noté de signe clinique précis et les poulets semblaient apparemment sains.

La contamination des poulets par Heterakis gallinarum était de 66 p. 100 cependant l'histomonose est très rare, et ce protozoaire a été trouvé dans une seule des lésions cæcales observées.

\section{Remerciements}

Nos plus vifs remerciements sont adressés à Monsieur le Docteur A. RAFYI pour les précieux conseils qu'il a bien voulu nous donner dans la conduite de cette étude.

\section{SUMMARY}

\section{Prevalence of helminths in poultry in Iran}

The authors investigated systematically helminths parasitizing hundred chickens of yarious origins.

They give the list of found parasites and indicate those pointed out for the first time in Iran. 


\section{RESUMEN}

Frecuencia de Ios helmíntos en las aves de corral en Iran

Los autores sistemáticamente buscaron los helmintos albergados por cien pollos de varias origenes.

Dans la lista de los parásitos encontrados, al precisar los que se señalan por primera vez en Iran.

\section{BIBLIOGRAPHIE}

1. MIRZAYANS (A.), NIAK (A.). The incidence of species of the genus Capillaria Zeder 1800 (Nematode) in chickens in Iran. Brit. vet. J., 1970, 126 (1): i-ii.

2. TAYLOR (E. L.). Field experiments on immunity of lambs to parasitic gastritis caused by mixed infection of trichostrongylid nematodes. J. Helminth, 1934, 12: 134-169.

3. ZARRINE (K.). Cestodes of industrial poultry in Teheran area (in Persian). Vet. Med. Diss. Teheran Univ., 1964, 35 p. 